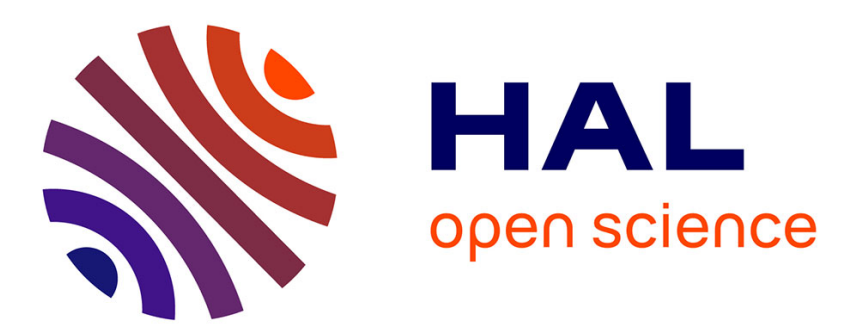

\title{
Numerical modelling of thermomechanical processes related to bulk forming operations
}

\author{
M. Schönauer, T. Rodic, D. Owen
}

\section{To cite this version:}

M. Schönauer, T. Rodic, D. Owen. Numerical modelling of thermomechanical processes related to bulk forming operations. Journal de Physique IV Proceedings, 1993, 03 (C7), pp.C7-1199-C7-1209. 10.1051/jp4:19937185 . jpa-00251817

\section{HAL Id: jpa-00251817 https://hal.science/jpa-00251817}

Submitted on 1 Jan 1993

HAL is a multi-disciplinary open access archive for the deposit and dissemination of scientific research documents, whether they are published or not. The documents may come from teaching and research institutions in France or abroad, or from public or private research centers.
L'archive ouverte pluridisciplinaire HAL, est destinée au dépôt et à la diffusion de documents scientifiques de niveau recherche, publiés ou non, émanant des établissements d'enseignement et de recherche français ou étrangers, des laboratoires publics ou privés. 


\title{
Numerical modelling of thermomechanical processes related to bulk forming operations
}

\author{
M. SCHÖNAUER*, T. RODIČ ${ }^{* * *}$ and D.R.J. OWEN* \\ * Institute for Numerical Methods in Engineering, University College of Swansea, Singleton Park, Swansea \\ SA2 8PP, Wales, U.K. \\ ${ }_{* *}$ Faculty for Natural Science and Technology, University of Ljubljana, Aškerčeva 20, 61000 Ljubljana, \\ Slovenia
}

\begin{abstract}
A thermomechanically sound, decoupled model, numerically unified for cold and hot bulk metal forming is cast into a finite element formulation. The mechanical module is based on elasto(visco)plastic material costitutive laws and general interface frictional contact conditions with the assumption of rigid tools. Introduction of a logarithmic strain based finite strain model in the contex of a geometrically nonlinear assumed strain method characterizes the numerical treatment of incompressibility at large deformations. A non-associated slip rule is employed in the contex of the plasticity theory of friction, with nonlinear master segment geometry describing the contact kinematics. Consistent linearisation in all aspects of algorithmic development provides robust and quadratically convergent solutions. This is shown in 2-D plane strain and axisymmetric numerical examples, where simulations of both cold and hot bulk metal forming processes are performed.
\end{abstract}

\section{INTRODUCTION}

The use of numerical simulations in the industrial design and optimization of metal forming processes has increased which has been made possible by the developing maturity of the necessary theoretical formulations and associated numerical algorithms together with the rapid improvement in computer hardware and software capabilities. The most efficient and reliable simulations rely on finite element based techniques, also utilized in the present work, which are well established as a powerful numerical framework for solving the complex thermo-physical interplay between the various factors encountered in forming operations. It is of utmost importance for a numerical analyst to have a clearly defined goal before incorporating qualitatively stutable models in a computer code, as well as for a forming engineer to be aware of both the level of approximation of these models and the computational capabilities of the associated numerical algorithms. The aim of this work is to develop a general and upgradable model for bulk forming operations which includes elastic response and which covers both cold and hot temperature regimes in a numerically unified manner. Such a model can then also be used, within the same area of application, for sheet metal forming, where the requirements of element technology are less demanding.

\section{MODELLING OF METAL FORMING PROCESSES}

The mechanical and thermal aspects of metal deformation during forming processes are extremely complex as a result of the many thermo-physical phenomena that interact, especially in high temperature regimes. The five balance laws of the theory of continuum physics ${ }^{[1,2]}$ provide a starting point for description of the coupled thermomechanical behaviour of a body. A set of constitutive 
equations, which imposes additional restrictions on the relationships between the unknowns in these laws, must be introduced into the model to represent the material behaviour under simulated loading conditions. The theory of continuum physics also provides the basic principles which assist in the construction of particular constitutive relations. These principles can be summarized by eight axioms ${ }^{[1,2]}$ and they provide a selection rule for differentiating dependant constitutive variables from the independent ones. They attribute every phenomenon to the independant constitutive variables of motion and temperature, from which the dependant variables; stress $\sigma$, strain $\epsilon$, heat flux $q$, specific entropy $s$, etc. can be derived. Therefore, whichever constitutive model is adopted, its independent constitutive variables must be motion (displacements), and temperature. Before particular constitutive models are investigated, an overall solution strategy for this coupled problem should be defined. Generally, discretization will provide two sets of equations which must be temporally integrated:

$$
\begin{gathered}
F_{M E C}(X(t), T(t))=0 \\
F_{T E R}(X(t), T(t))=0
\end{gathered}
$$

where $\boldsymbol{F}_{M E C}$ states the equilibrium conditions in the discretised body ("mechanical equilibrium") and $\boldsymbol{F}_{T E R}$ represents the coupled heat conduction equation ("thermal equilibrium") at any time $t$, while the column matrices $\boldsymbol{X}$ and $\boldsymbol{T}$ refer to the coordinates and temperatures of the nodal points in the discretised domain. To advance the solution in time from the known solution at time $\bar{t}$ to $\bar{t}+\Delta t$, evaluation of the appropriate gradient matrices is required. If the mechanical and thermal equilibrium are treated simultaneously by a Newton type procedure, the off-cliagonal gradient matrices $\partial F_{M E C} / \partial T$ and $\partial F_{T E R} / \partial X$ must be evaluated. Since this is a difficult task and the resulting computational procedure involves a large number of numerical operations, an alternative two-step scheme, preferred by the majority of analysts $\left(\right.$ e.g. $\left.{ }^{[3,4]}\right)$, is utilized:

$$
\begin{array}{cl}
1^{s t} \text { step: } & F_{M E C}\left(X_{i+1}, T_{i}\right)=0 \\
2^{\text {nd }} \text { step: } & F_{T E R}\left(X_{i+1}, T_{i+1}\right)=0
\end{array}
$$

Thus, equations (2-3) are solved separately and coupling variables are then suitably exchanged at appropriate stages of analysis.

\subsection{Heat conduction model}

Transfer of energy during forming operations, in the general form expressed by (5), is a complex process. At the same time during which heat is removed from the workpiece due to contact with the colder dies, by radiation or by water cooling, heat is also generated by dissipative mechanical mechanisms such as plastic work and friction. The resulting temperature changes in the workpiece, in turn, affect the material response to the imposed deformation. In the present model, a thermodynamically sound derivation is adopted ${ }^{[5,6]}$. Based on the conservation of energy and entropy inequality laws:

$$
\begin{gathered}
\rho \dot{e}=\sigma_{i j} \dot{\epsilon}_{i j}-q_{k, k}+q_{v} \\
\rho T \dot{s} \geq q_{k, k}-(1 / T) q_{i} T_{, i}+q_{v} \geq 0
\end{gathered}
$$

the following coupled heat conduction differential equation is obtained:

$$
-\operatorname{div} q+q_{v}+\xi \dot{w}^{i}=\rho\left(c \dot{T}+\dot{s}_{T} T\right)
$$

where $\rho$ is the mass density, $q_{v}$ is the heat supply per unit volume per unit time, $T$ is the absolute temperature, $\xi$ is the portion of inelastic work $w^{i}$ which is converted into heat, $c$ is the specific heat capacity, and $\dot{s}_{T}$ the rate of specific entropy at constant temperature. Locally 
the thermomechanical coupling arises through $w^{i}=\sigma: \epsilon^{i}$, where $\epsilon^{i}$ is the inelastic part of the strain tensor, and through $\dot{s}_{T}$, which has to be derived from the free energy function $\phi$ for the particular material, but its overall contribution to equation (8) can be usually neglected in numerical simulations.

Box 1. Elasto-plastic material model

(1) Additive strain decomposition:

$$
\epsilon=\epsilon^{e}+\epsilon^{p}
$$

(2) Elastic response:

$$
\begin{gathered}
\boldsymbol{\sigma}=\mathbf{H}: \boldsymbol{\epsilon}^{\mathrm{e}} \\
\mathbf{H}=\frac{\partial^{2} W\left(\boldsymbol{x}, \boldsymbol{\epsilon}^{e}\right)}{\partial \epsilon^{e^{2}}}=\lambda \mathbf{1} \otimes \mathbf{1}+2 \mu \mathbf{I}
\end{gathered}
$$

(3) Yield function:

$$
F\left(\sigma, \bar{\epsilon}^{p}\right)=f(\sigma)-\sigma_{Y}\left(\bar{\epsilon}^{p}\right)
$$

(4) Flow rule:

$$
\dot{\boldsymbol{\epsilon}}^{p}=\dot{\gamma} \frac{\partial Q}{\partial \sigma}
$$

(5) Von-Mises associated plasticity:

$$
f(\boldsymbol{\sigma})=\bar{\sigma} ; Q=F ; \frac{\partial F}{\partial \boldsymbol{\sigma}}=\sqrt{3 / 2} \frac{\operatorname{dev} \boldsymbol{\sigma}}{\|\operatorname{dev} \boldsymbol{\sigma}\|}
$$

(6) Hardening law:

$$
\dot{\sigma}_{Y}=\frac{\partial \sigma_{Y}}{\partial \bar{\epsilon}^{p}} \dot{\epsilon^{p}}=H^{\prime} \sqrt{2 / 3 \dot{\epsilon}^{p}: \dot{\epsilon}^{p}}
$$

(7) Loading-unloading conditions:

$$
\dot{\gamma} \geq 0, F \leq 0, \dot{\gamma} F=0
$$

(8) Consistency condition:

$$
\dot{\gamma} \dot{F}=0
$$

Box 2. Elasto-viscoplastic material model

(1) Additive strain rate decomposition:

$$
\dot{\boldsymbol{\epsilon}}=\dot{\boldsymbol{\epsilon}}^{e}+\dot{\epsilon}^{v p}
$$

(2) Elastic response:

$$
\dot{\epsilon}^{e}=\mathbf{H}^{-1}: \dot{\sigma}
$$

(3) Flow rule:

$$
\dot{\boldsymbol{\epsilon}}^{v p}=\Upsilon\langle\Phi(\bar{\sigma})\rangle \sqrt{3 / 2} \frac{\operatorname{dev}(\boldsymbol{\sigma})}{\|\operatorname{dev}(\boldsymbol{\sigma})\|}
$$

(4) $\Upsilon$ and $\Phi$ from interpolation functions:

$$
\begin{gathered}
\Upsilon=\left[\frac{1}{A_{1} A_{2} A_{3}} \exp \left(m_{1} T\right) \bar{\epsilon}^{v p\left(-m_{2}\right)}\right]^{\left(1 / m_{3}\right)} \\
\Phi=\left(\frac{\bar{\sigma}}{\Gamma_{f o}}\right)^{\left(1 / m_{3}\right)} \\
\underline{\text { (ii) Sellars-Tegart }} \\
\Upsilon=C \exp \left[\frac{-Q}{R(T+273)}\right] \\
\Phi=[\sinh (\alpha \bar{\sigma})]^{n} \\
\frac{(\text { iii }) \text { ALSPEN }}{\Phi=[c(T)]^{(-1 / m(T))}\left[\alpha\left(\bar{\epsilon}^{v p}\right)+\alpha_{0}\right]^{(-n(T) / m(T))}} \\
\Phi=(\bar{\sigma})^{(1 / m(T))}
\end{gathered}
$$

\subsection{Mechanical constitutive model}

In addition to the elastic response, the mechanical constitutive relation is usually described by expressing the effective stress $\bar{\sigma}$, in metal forming terms usually called the flow stress, as a function of all other factors; $\bar{\sigma}=f\left(\bar{\epsilon}^{p}, \dot{\bar{\epsilon}}^{p}, T, S\right)$, where $\bar{\epsilon}^{p}$ and $\dot{\bar{\epsilon}}^{p}$ are respectively the effective plastic strain and effective plastic strain-rate, $T$ is the temperature and $S$ stands for all microstructure related factors, usually represented by material parameters (constants). In the present work, two constitutive relations, depending on the temperature regime, are considered. At low (room) temperature, the flow stress of most metals is practically strain-rate independant, thus only strainhardening (softening) behaviour is assumed: $\bar{\sigma}=f\left(\bar{\epsilon}^{p}\right)$. This leads to the well known theory of rate-independent plasticity, It is summarized in Box 1. for the associated Von-Mises flow rule which closely represents the behaviour of metals. Only isotropic strain hardening is assumed.

On the other hand for high temperature, the flow stress is assumed to be rate-dependant with possible strain hardening: $\bar{\sigma}=f\left(\dot{\bar{\epsilon}}^{v p}, \bar{\epsilon}^{v p}, T\right)$. This concept leads to rate-dependent theories of visco-plasticity. The so called Perzyna form of viscoplasticity, suitable for computational treatment, is summarized in Box 2. for an associated Von-Mises flow rule. For this case, it can be shown that $\dot{\bar{\epsilon}}^{v p}=\Upsilon \Phi$; thus the product of scalar functions, $\Upsilon$ and $\Phi$ uniquely defines the magnitude or 
intensity of viscoplastic flow, while the gradient $\partial F / \partial \sigma$ determines its direction only. This fact gives rise to the approach proposed by Rodic ${ }^{[5]}$, also pursued here, where the crucial idea is to express $\Upsilon$ and $\Phi$ in terms of so called high temperature interpolation functions for the flow stress. Metallurgical analysts have suggested several empirical expressions for these functions in order to fit experimental data obcained from tests on various materials under different conditions. Three expressions from the literature, chosen for numerical implementation in the present model are:

(1) Hajduk expression. The flow stress $\bar{\sigma}$ is assumed to be expressed in the following form:

$$
\bar{\sigma}=K_{f o} K_{T} K_{\bar{\epsilon}} K_{\dot{\bar{\epsilon}}}
$$

where the three coefficients are functions of the form:

$$
K_{T}=A_{1} \exp \left(-m_{1} T\right) ; K_{\bar{\epsilon}}=A_{2} \bar{\epsilon}^{m_{2}} ; K_{\dot{\bar{\epsilon}}}=A_{3} \dot{\bar{\epsilon}}^{m_{3}}
$$

where $A_{i}$ and $m_{i}$ are material constants to be determined.

(2) Sellars-Tegart expression. This is based on the following interpolation equation:

$$
Z=\dot{\bar{\epsilon}} \exp \left[\frac{Q}{R(T+273)}\right]=C[\sinh (\alpha \bar{\sigma})]^{n}
$$

where $Z$ is the Zener-Hollomon parameter, $Q$ is an activation energy usually independent of temperature and in many cases also inclependent of strain, $R$ is the gas constant $8.31 \mathrm{~J} / \mathrm{molK}, T$ is the temperature in degrees Celsius, while $C, \alpha$ and $n$ are material constants.

(3) ALSPEN expression ${ }^{[7,8]}$ : This expression is found to cover closely the properties of some aluminium alloys by fitting experimental curves in the form:

$$
\bar{\sigma}=c(T)\left(\alpha+\alpha_{0}\right)^{n(T)} \dot{\bar{\epsilon}}^{m(T)}
$$

Coefficients $c(T), n(T)$ and $m(T)$ are described in the references, $\alpha_{0}$ is a constant taken to be 0.001 , and $d \alpha=d \bar{\epsilon}^{v p}$ for temperatures below the onset limit $T_{0} \approx 700 K$, otherwise $d \alpha=0$.

Since practically $\bar{\epsilon}=\bar{\epsilon}^{v p}, \dot{\bar{\epsilon}}=\dot{\bar{\epsilon}}^{v p}$, parameters $\Upsilon$ and $\Phi$ can be obtained, as shown in Box 2., for all three interpolation functions. In all three cases, the yield stress is taken to be zero, thus the assumption is made that some part of the strain is always inelastic. It should be noted that this concept fits into the general framework of strain decomposition, olstained by using the plastic flow potential $\psi$ and free energy potential $\phi$, where $\epsilon^{v p}, \sigma$, the tensor of internal variables $q$ which describes the state of the microstructure, and the "thermodynamic force" $Q$ conjugate to the rate of change of $q$, are defined in the following form:

$$
\dot{\epsilon}^{v p}=\frac{\partial \psi}{\partial \sigma} ; \sigma=\frac{\partial \phi}{\partial \epsilon^{e}} ; \dot{q}=\frac{\partial \psi}{\partial Q} ; \boldsymbol{Q}=\frac{\partial \phi}{\partial q}
$$

The relevant potentials in the present model can be recovered from the general expressions proposed by $\mathrm{Kim}$ and $O \operatorname{den}[9,10]$ for isotropic materials.

The models in Boxes 1. and 2. are valid for the small strain case, but their inclusion in the finite strain model adopted in the present work is directly obtained by replacing the pair $\{\sigma, \epsilon\}$ with the conjugate pair $\{\boldsymbol{T}, \ln U\}$, where logarithmic stretches and the conjugate rotated stress are employed as strain and stress measures. This is justified, since the effects of finite strains defined in this way appear only at the kinematic level. For a detailed discussion of the adopted finite strain model, one is referred to the contributions of Peric and co-workers ${ }^{[1-13]}$, which is summarized here in Box 3. 
Box 3. Finite strain model

(1) Polar decomposition of $F$ :

$$
F=\frac{\partial x}{\partial X}=R U
$$

(2) Computation of logarithmic stretches:

$$
\begin{gathered}
C=F^{t} \boldsymbol{F}=\sum_{I=1}^{3} C_{(I)} N^{(I)} \otimes N^{(I)} \\
\ln U=\sum_{I=1}^{3} \ln \lambda_{(I)} N^{(I)} \otimes N^{(I)} ; \lambda_{(I)}=C_{(I)}^{I / 2}
\end{gathered}
$$

(3) Strain energy function:

$$
W=\mu \sum_{I=1}^{3}\left(\ln \lambda_{(I)}^{e}\right)^{2}+\frac{1}{2} \lambda\left(\ln J^{e}\right)^{2}
$$

(4) Multiplicative decomposition of $F$ :

$$
F=F^{e} F^{p}
$$

(5) Elasto-plasticity, elasto-viscoplasticity:

in Boxes 1. and 2. with $\epsilon=\ln U, \sigma=T$

\subsection{Tool-workpiece interface model}

The assumption of rigid tools is made in the present model, which is justified in most metal forming operations where the deformability of the workpiece is several orders of magnitude larger than for the tools. A crucial issue in the numerical modelling of tool-workpiece interface conditions is an appropriate frictional contact model. Unfortunately, because of its microscopic nature, friction cannot be modelled precisely in the numerical analysis of macroscopic metal forming processes. The frictional resistance to slicling is therefore simulated by assuming mathematical relationships between contact tractions and other contact related variables. In the present work, a quasi static type of friction, defined by Oden and Martins ${ }^{[14]}$, is considered while the micromechanical model is provided by Avitzur and Nakamura ${ }^{[15]}$ and approximated with a combination of Coulomb and constant shear friction law. This is incorporated within the so called plasticity theory of friction ${ }^{[16-18]}$, which is introduced in a specific nonlinear kinematic framework. The main feature of this formulation is the introduction of a nonlinear coordinate $\xi$ along the master (tool) surface, which is discretized utilizing two geometric primitives: straight segments and circular arc segments. It should be noted that in the latter case, gap decomposition differs from that of Curnier ${ }^{[18]}$. The general case is shown in Fig. 1 where $S$ denotes a slave node on the deformable workpiece surface, $M$ its closest projection on the master surface, while $M_{0}$ is the initial point of uninterrupted contact for S. The basic expressions for the above defined aspects of frictional contact are summarized in Box 4.

Box 4. Frictional contact model

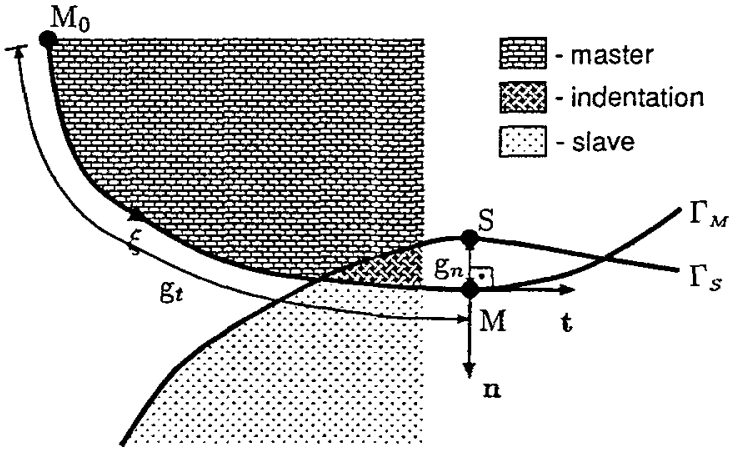

Fig.1. Master-slave contact line kinematics
(1) Kinematic framework:

$$
\begin{aligned}
& \mathrm{g}_{n}=\mathrm{g}_{n} n ; \mathrm{g}_{n}=\left(\boldsymbol{x}_{s}-\boldsymbol{x}_{M}\right) \cdot \boldsymbol{n} \\
& \mathrm{g}_{t}=\mathrm{g}_{t} t ; \mathrm{g}_{t}=\xi\left(\boldsymbol{x}_{M}\right)-\xi\left(\boldsymbol{x}_{M_{0}}\right)
\end{aligned}
$$

(2) Stick-slip decomposition:

$$
\mathrm{g}_{n}=\mathrm{g}_{n}^{e} \quad ; \quad \mathrm{g}_{t}=\mathrm{g}_{t}^{e}+\mathrm{g}_{t}^{p}
$$

(3) Stick response:

$$
\sigma_{n}=c_{n} g_{n}^{e} \quad ; \quad \tau=c_{t} g_{t}^{e}
$$

(4) Slip criterion:

$$
\begin{gathered}
F_{S L}\left(\sigma_{n}, \tau\right)=|\tau|-\tau_{S L}=0 \\
\tau_{S L}=\left\{\begin{array}{cc}
\mu\left|\sigma_{n}\right| & \text { for }\left|\sigma_{n}\right|<\tau_{m} / \mu \\
\tau_{m} & \text { for }\left|\sigma_{n}\right| \geq \tau_{m} / \mu
\end{array}\right.
\end{gathered}
$$

(5) Nonassociated slip rule:

$$
\dot{\mathrm{g}}_{t}^{p}=\dot{\gamma}_{s L} \frac{\partial Q_{s L}}{\partial \sigma^{c}} \quad ; \quad Q_{s L}=|\tau|
$$




\section{NUMERICAL FINITE ELEMENT FORMULATION}

The models defined in Section 2. must be embedded in a variational principle suitable for a finite element formulation. The crucial task is to develop an element which provides high accuracy with relatively coarse meshes, and which treats properly the incompressible nature of the plastic flow, i.e. does not exhibit "spurious locking". In this respect, the geometrically nonlinear assumed strain method, proposed by Simo and Armero ${ }^{[19]}$, is introduced in the context of the present finite strain model and is briefly described. Inclusion of the frictional contact model in the resulting formulation is subsequently discussed, while the straightforward discretization of the coupled heat conduction model is mentioned at the end of the section.

\subsection{Geometrically nonlinear enhanced strain method}

The essential steps in the formulation and discretization of the problem are presented in Box 5 . and more details can be found in the original references on the enhanced strain method ${ }^{[19]}$ and the logarithmic finite strain mode $]^{[1-13]}$.

Box 5. Enhanced strain model

(1) Assumed deformation gradient:

$$
\boldsymbol{F}=1+\operatorname{Grad} \boldsymbol{u}+\tilde{H}
$$

(2) Enhanced spatial variational equations:

$$
\begin{gathered}
\int_{\Omega} \tilde{\nabla}(\delta \boldsymbol{u}): \tilde{\boldsymbol{\tau}}(\boldsymbol{u}, \tilde{\boldsymbol{h}}) d \Omega-G_{e z t}(\delta \boldsymbol{u})=0 \\
\int_{\Omega} \delta \tilde{\boldsymbol{h}}:[\tilde{\boldsymbol{\tau}}(\boldsymbol{u}, \tilde{\boldsymbol{h}})-\tau] d \Omega=0 \\
\int_{\Omega} \delta \boldsymbol{\tau}: \tilde{\boldsymbol{h}} d \Omega=0 \\
\tilde{\boldsymbol{h}}=\tilde{\boldsymbol{H}} \boldsymbol{F}^{-1} \\
\tilde{\boldsymbol{\tau}}(\boldsymbol{u}, \tilde{\boldsymbol{h}})=\tilde{\boldsymbol{R}} \frac{\partial \widehat{W}(\ln \tilde{U})}{\partial(\ln \tilde{U})} \tilde{\boldsymbol{R}}^{t}
\end{gathered}
$$

(3) Finite element interpolation of $\tilde{H}$ :

$$
\tilde{H}_{\mathrm{e}}=\sum_{t=1}^{\mathrm{N} E \mathrm{NH}} \alpha_{I}^{e} \otimes Q_{I}^{e} \quad ; \quad\left\langle P_{e}, \tilde{H}_{e}\right\rangle_{L_{z}}=0
$$

(4) Element linearized operators:

$$
\begin{gathered}
\tilde{\nabla}^{s} u_{e}^{h}=b_{d}\left(\widetilde{\operatorname{grad}} N_{e}^{I}\right) d_{e} ; \tilde{h}^{g}=b_{a}\left(q_{e}^{I}\right) \alpha_{e} \\
\tilde{\nabla} u_{e}^{h}=g_{d}\left(\widetilde{\operatorname{grad}} N_{e}^{I}\right) d_{e} ; \tilde{h}=g_{a}\left(q_{e}^{I}\right) \alpha_{e} \\
\widetilde{\operatorname{grad}} N_{e}^{I}=F_{e}^{-t} \operatorname{Grad} N_{e}^{I} ; q_{e}^{I}=F_{e}^{-t} Q_{I}^{e}
\end{gathered}
$$

(5) Element residual equations:

$$
\begin{gathered}
\boldsymbol{r}_{d}=\int_{\Omega_{e}} b_{d}^{t}: \tilde{\tau} d \Omega_{e}-f^{e x t} \\
\boldsymbol{r}_{a}=\int_{\Omega_{e}} \boldsymbol{b}_{\alpha}^{t}: \tilde{\boldsymbol{\tau}} d \Omega_{e}
\end{gathered}
$$

(6) Newton's method of linearization:

$$
\begin{gathered}
\boldsymbol{k}_{d d} \Delta d_{e}+\boldsymbol{k}_{a d}^{t} \Delta \alpha_{e}=-\boldsymbol{r}_{d} \\
\boldsymbol{k}_{a d} \Delta d_{e}+\boldsymbol{k}_{a \alpha} \Delta \alpha_{e}=-\boldsymbol{r}_{a} \\
\boldsymbol{k}_{d d}=\int_{\Omega_{e}} \boldsymbol{b}_{d}^{t}\left(\mathbf{h}_{\mathrm{E}(\mathrm{V}) \mathrm{P}}-\mathbf{m}\right) \boldsymbol{b}_{d} d \Omega_{e}+\int_{\Omega_{e}} \boldsymbol{g}_{d}^{t} \hat{\sigma} \boldsymbol{g}_{d} d \Omega_{e} \\
\boldsymbol{k}_{a d}=\int_{\Omega_{e}} \boldsymbol{b}_{a}^{t}\left(\mathbf{h}_{\mathrm{E}(\mathrm{V}) \mathrm{P}}-\mathbf{m}\right) \boldsymbol{b}_{d} d \Omega_{e}+\int_{\Omega_{e}} \boldsymbol{g}_{a}^{t} \hat{\boldsymbol{\sigma}} \boldsymbol{g}_{d} d \Omega_{e} \\
\boldsymbol{k}_{a a}=\int_{\Omega_{e}} b_{a}^{t}\left(\mathbf{h}_{\mathrm{E}(\mathrm{V}) \mathrm{P}}-\mathbf{m}\right) \boldsymbol{b}_{a} d \Omega_{e}+\int_{\Omega_{e}} \boldsymbol{g}_{a}^{t} \hat{\boldsymbol{\sigma}} \boldsymbol{g}_{a} d \Omega_{e}
\end{gathered}
$$

(7) Static condensation on the element level:

$$
\begin{gathered}
\tilde{k}^{e} \Delta d_{e}=\tilde{\boldsymbol{r}}^{\mathrm{e}} \\
\Delta \alpha_{e}=-\boldsymbol{k}_{a a}^{-1}\left(\boldsymbol{r}_{a}+k_{a d} \Delta d_{\mathrm{e}}\right) \\
\tilde{k}^{e}=\boldsymbol{k}_{d d}-\boldsymbol{k}_{a d}^{t} \boldsymbol{k}_{a a}^{-1} k_{a d} \\
\tilde{\boldsymbol{r}}^{\mathrm{e}}=\boldsymbol{r}_{d}-k_{a d}^{t} \boldsymbol{k}_{a a}^{-1} \boldsymbol{r}_{a}
\end{gathered}
$$

(8) Global displacement equations:

$$
\begin{aligned}
& \tilde{K} \Delta d=-\bar{G}
\end{aligned}
$$

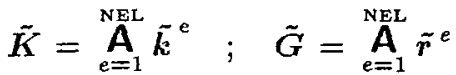

The crucial idea is to assume the displacement gradient $H$ to be a sum of the compatible part Grad $\boldsymbol{u}$ and the enhanced part $\tilde{\boldsymbol{H}}$, which also implies multiplicative decomposition $\boldsymbol{F}=\tilde{\boldsymbol{F}} \boldsymbol{F}_{\boldsymbol{x}}$ where $\tilde{F}=1+\tilde{H} F_{x}^{-1}$ and $F_{\mathfrak{x}}=\partial x / \partial X$ represent respectively the enhanced and compatible contributions. It is employed in a suitable form of the 3-field $\mathrm{Hu}$-Washizu variational principle, which reduces to a 2 -field ( $\boldsymbol{u}$ and $\tilde{\boldsymbol{h}}$ ) form after the stress field $\tau$ is effectively eliminated by enforcing $L_{2}$ orthogonality between the approximative spaces of stress and enhanced displacement gradient. 
The two remaining equations are numerically discretized in the usual finite element manner. Standard material and geometrical parts of the element stiffness matrix are recovered consistent with the finite strain model employed and a radial return algorithm used in the integration of the constitutive equations. In that context, the consistent elasto-(visco)plastic tangent operator for small strain situations, $\boldsymbol{h}_{E(V) P}$, is directly employed. Interelement discontinuity of the enhanced part of the displacement gradient enables static condensation on the element level and hence global displacement-type equations are obtained. These are solved in the usual incremental-iterative manner where quadratic convergence of the algorithm is ensured by consistent linearization.

It should be noted that although locking is avoided in simulations using the resulting formulation, the opposite effect of hourglassing may be encountered, depending on a particular choice of the reference configuration.

\subsection{Frictional contact problem}

Contribution from the frictional contact model presented in Box 4., must be added to the overall equilibrium equation. Referring to a single node in contact with a master segment, the relevant finite element expressions can be summarised in Box 6., where general component notation is employed for convenience.

Box 6. F.E. formulation for a contact node

(1) Notational considerations:

$$
\sigma^{c}=\left(\tau, \sigma_{n}\right) ; \mathrm{g}=\left(\mathrm{g}_{t}, \mathrm{~g}_{n}\right) ; \boldsymbol{u}^{\mathrm{NOD}}=\left(u_{\boldsymbol{x}}^{\text {NOD }}, u_{y}^{\text {NOD }}\right)
$$

(2) Associated area and force:

$$
A=\frac{1}{2}\left(\left\|x^{\mathrm{S}}-x^{\mathrm{N} 1}\right\|+\left\|x^{\mathrm{S}}-x^{\mathrm{N}^{2}}\right\|\right) t^{\mathrm{S}} \quad ; \quad f^{c}=A \sigma^{c}
$$

(3) Variational formulation and residual contribution:

$$
\tilde{W}_{c}=f_{k}^{c} \delta g_{k}=f_{k}^{c} \frac{\partial g_{k}}{\partial u_{i}^{\mathrm{s}}} \delta u_{i}^{\mathrm{s}} \quad ; \quad r_{i}^{\mathrm{s}}=f_{k}^{c} \frac{\partial g_{k}}{\partial u_{i}^{\mathrm{s}}}
$$

(4) Stiffness contribution:

$$
\begin{gathered}
k_{i j}^{\mathrm{ss}}=\frac{\partial r_{i}^{\mathrm{s}}}{\partial u_{j}^{\mathrm{s}}}=\left[\frac{\partial A}{\partial u_{j}^{\mathrm{s}}} \sigma_{k}^{c}+A \frac{\partial \sigma_{k}^{c}}{\partial g_{l}} \frac{\partial \mathrm{g}_{l}}{\partial u_{j}^{\mathrm{s}}}\right] \frac{\partial g_{k}}{\partial u_{i}^{\mathrm{s}}}+\frac{\partial}{\partial u_{j}^{\mathrm{s}}}\left(\frac{\partial g_{k}}{\partial u_{i}^{\mathrm{s}}}\right) f_{k}^{c} \\
k_{i j}^{\mathrm{s} \mathrm{N}^{1(2)}}=\frac{\partial r_{i}^{\mathrm{s}}}{\partial u_{j}^{\mathrm{N} 1(2)}}=\frac{\partial A}{\partial u_{j}^{\mathrm{N} 1(2)}} \sigma_{k}^{c} \frac{\partial \mathrm{g}_{k}}{\partial u_{i}^{\mathrm{s}}}
\end{gathered}
$$

(5) Stick and slip operators:

$$
\frac{\partial \sigma^{c}}{\partial \mathrm{g}}=\left\{\begin{array}{cc}
D_{S T}=\left[\begin{array}{cc}
c_{t} & 0 \\
0 & c_{n}
\end{array}\right] & \text { if } F_{S L}<0 \\
D_{s L}=\left[\begin{array}{cc}
0 & c_{n} \operatorname{sign}(\tau) \frac{\partial F_{s L}}{\partial \sigma_{n}} \\
0 & c_{n}
\end{array}\right] & \text { if } F_{S L}=0
\end{array}\right.
$$

The area $A$, associated with the slave node $\mathrm{S}$, is defined by means of two neighbouring nodes N1 and $\mathrm{N} 2$, and thickness $t^{\mathrm{s}}$. The virtual work variational principle is used to obtain the residual equation, the linearization of which provides the stiffness terms. Since a unilateral contact constraint is assumed, the position of tool (master) segments at the end of a time increment under consideration is known in advance. Thus, the second order gap variation is non-zero only in the case of contact with a nonlinear (circular arc) master segment. In contrast to the case of two deformable bodies, where the resulting terms are found to be essential to achieve quadratic convergence ${ }^{[0,21]}$, 
numerical tests have indicated that they can usually be neglected in the present model. The area variation can be easily obtained, and the thickness variation should also be considered for axisymmetric problems. The non-associated slip rule results in non-symmetric consistent moduli, which are written explicitly in matrix form for the 2-D case. Non-symmetry also arises through $\boldsymbol{k}^{\mathrm{SN} 1(2)}$, since $k^{\mathrm{N} 1(2) S}=0$. It should be noted that although a non-penetrating constraint $g_{n} \geq 0$ is not assumed, the resulting formulation is the same as if the constraint was imposed and introduced in a penalty form. Then, one would recover $c_{n}$ as 'a choice of the penalty parameter on physical grounds'[22].

\subsection{Coupled heat transfer equation}

The finite element discretization of the heat transfer equation is well known and can be found in the literature (e.g. ${ }^{[23]}$ ). The only additional step in discretization of the present equation (8) is inclusion of the coupling terms in the formulation of the quasi-stiffness matrix and quasi-force vector. This simple procedure is described in detail in ${ }^{[5]}$.

\section{NUMERICAL EXAMPLES}

4.1. Plane strain localization problem. Plane strain tensile test of a saturation hardeninglinear softening rectangular bar ${ }^{[19]}$ is considered as a severe numerical benchmark test. Due to symmetry, one quarter of the specimen is discretized, while the geometry and material properties are the same as in the reference. The excellent performance of the element in capturing physically well observed localization of plastic deformation along shear bands is shown in Fig.2., where the initial and distorted meshes for the various stages of the test are presented. The distribution of effective plastic strain in the necking region is provided in Fig.3.

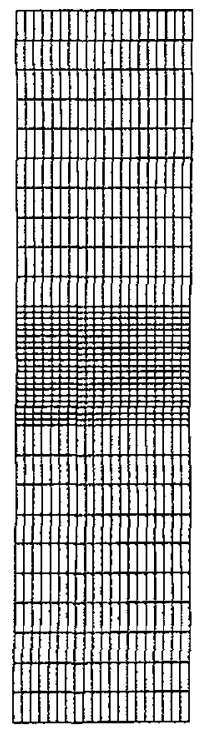

(a)

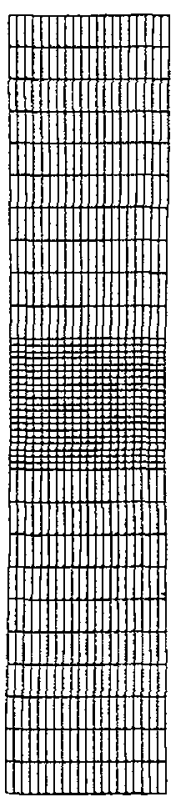

(b)

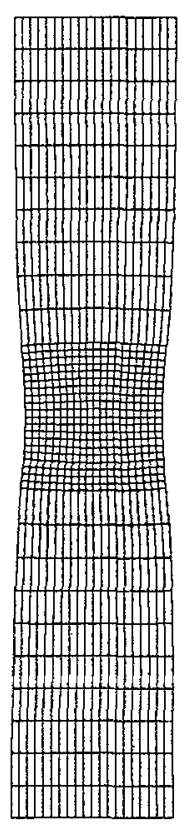

(c)

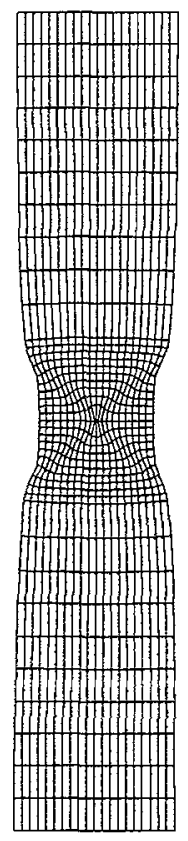

(d)

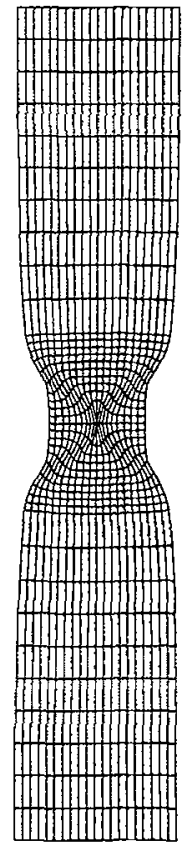

(e)

Fig.2. Plane strain tensile test of a rectangular bar: (a) initial configuration; deformed configuration at different stages of imposed displacement: (b) $\bar{u}=2.50$; (c) $\bar{u}=3.35$; (d) $\bar{u}=4.00$; (e) $\bar{u}=4.50$. 


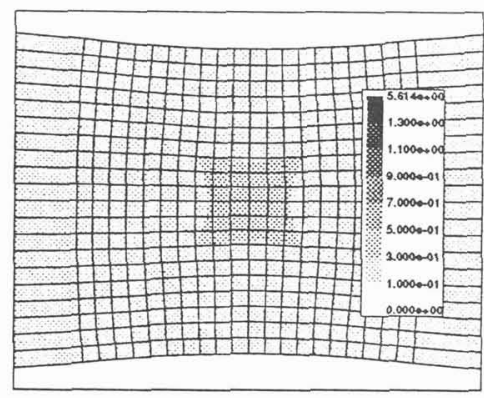

(a)

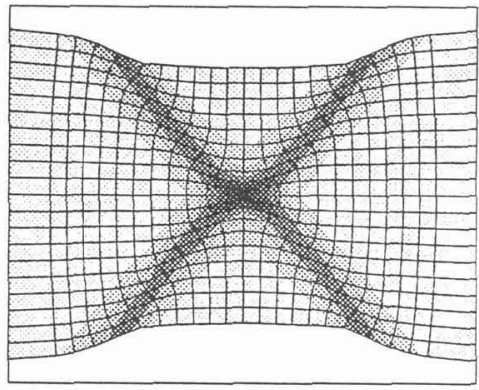

(c)

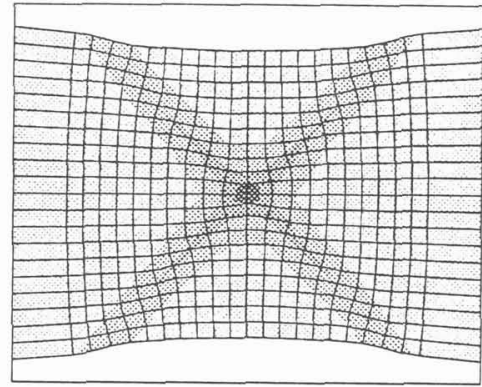

(b)

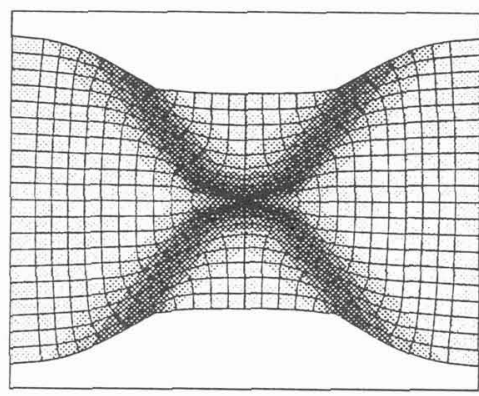

(d)

Fig.3. Plane strain tensile test of a rectangular bar. Effective plastic strain distribution in the necking region at different stages: (a) $\bar{u}=3.35$; (b) $\bar{u}=3.60$; (c) $\bar{u}=4.00$; (d) $\bar{u}=4.50$;

4.2. Plane strain cold rolling. This example of an aluminium strip cold rolling with $33.75 \%$ of height reduction has been considered experimentally by Al-Salehi et al. ${ }^{[24]}$, and numerically by Li \& Kobayashi ${ }^{[25]}$ (case 8), where a rigid-plastic finite-element formulation is employed. Due to the neglect of elastic response, such a "flow formulation" model is not capable of predicting residual stresses. However, it is not an obstacle in the present model, and this useful prediction is shown in Fig.4. The effective plastic strain distribution, which is in good agreement with ${ }^{[25]}$, is also depicted in Fig.5.
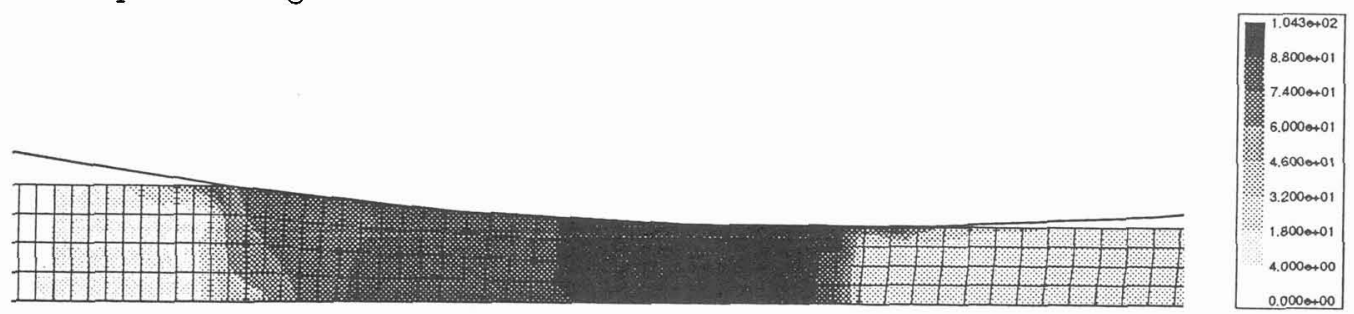

Fig.4. Plane strain cold rolling. Effective stress distribution
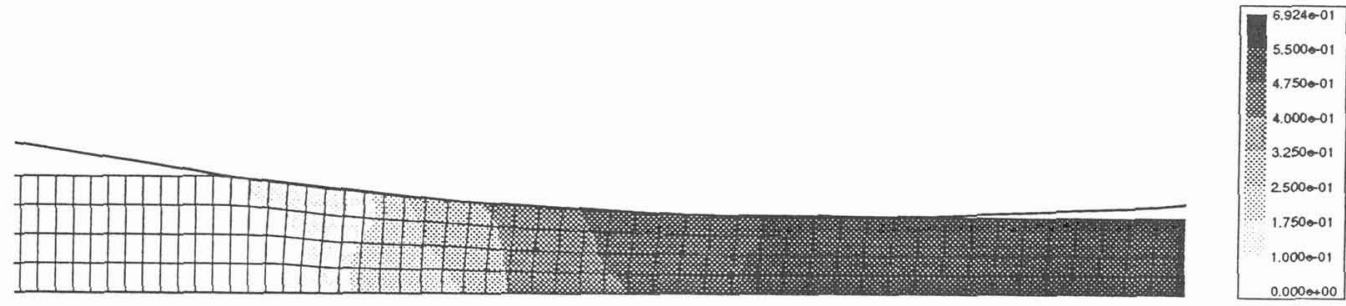

Fig.5. Plane strain cold rolling. Effective plastic strain distribution 
4.3. Plane strain hot forging. Plane strain conditions are assumed in the hot forging of the industrial component illustrated in Fig.6. The material elastic constants are $E=120000 \mathrm{~N} / \mathrm{mm}^{2}$, $\nu=0.3$, while the viscoplastic flow is assumed to follow the Sellars-Tegart interpolation function with material coefficients $C=1.2 \cdot 10^{12} \mathrm{~s}^{-1}, \alpha=1.12 \cdot 10^{-2} \mathrm{~mm}^{2} / \mathrm{N}, n=3.5, Q=352690 \mathrm{~J} / \mathrm{mol}$. Isothermal conditions $\left(T=1100^{\circ} C\right)$ and a Coulomb frictional law $(\mu=0.3)$ are assumed. The tool is discretized with a combination of circular arc and straight segments. Figure 6 . shows the initial and various deformed meshes together with effective plastic strain distribution. No remeshing is undertaken, hence some finite elements become severely distorted, prohibiting further advance of tool displacement at approx. $85 \%$ of the target value.

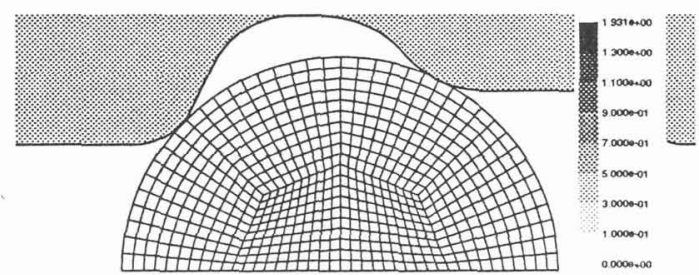

(a)

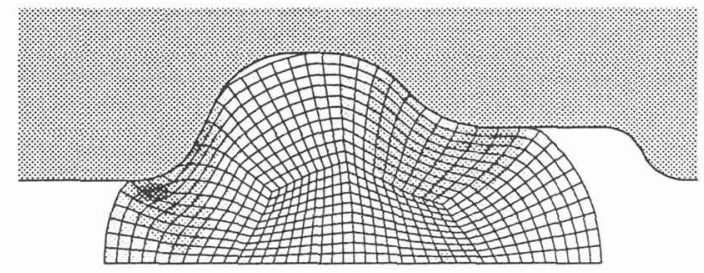

(b)

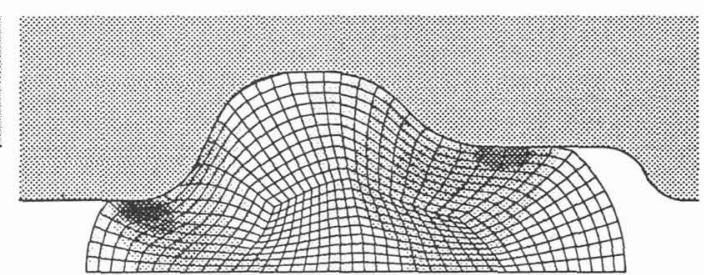

(c)

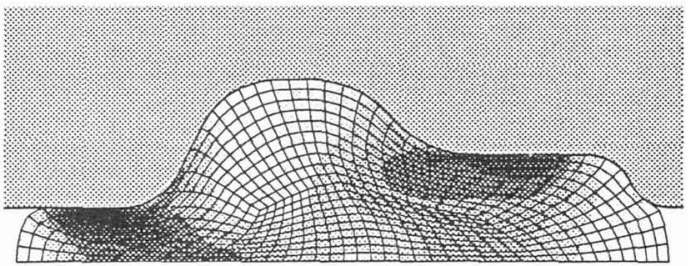

(d)

Fig.6. Plane strain hot forging. Effective plastic strain distribution for one half of the cross-section at different stages of tool displacement: (a) initial configuration; (b) $\bar{u}=2.5 \mathrm{~mm}$; (c) $\bar{u}=4.1 \mathrm{~mm}$; (d) $\bar{u}=6.9 \mathrm{~mm}$.

4.4. Axisymmetric hot spike forming. The simulation of this test is presented to assess the perfomance of the present axisymmetric element. A cylindrical billet $(r=7.5 \mathrm{~mm}, h=$ $20.0 \mathrm{~mm}$ ), with material properties as in example 4.3. and a combined friction law $(\mu=0.3$, $\tau_{m}=100 \mathrm{~N} / \mathrm{mm}^{2}$ ) is forged in an impression die containing a central cavity at assumed isothermal conditions $\left(T=1000^{\circ} \mathrm{C}\right)$. The numerical results are in excellent agreement with experimental observations regarding both the neutral point phenomenon and the flow patterns, where in the first stage (Fig.7.(a)-(c)) the height is reduced while in the second stage (Fig.7.(c)-(e)) it increases significantly.

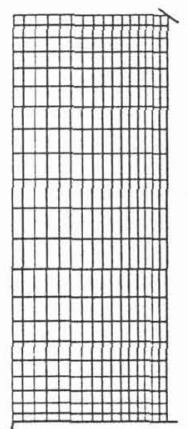

(a)

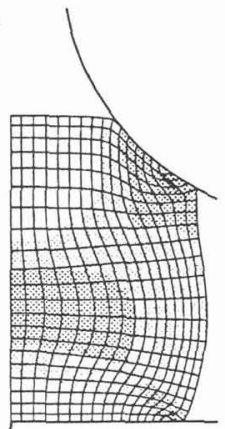

(b)

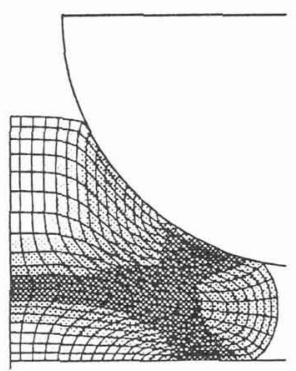

(c)

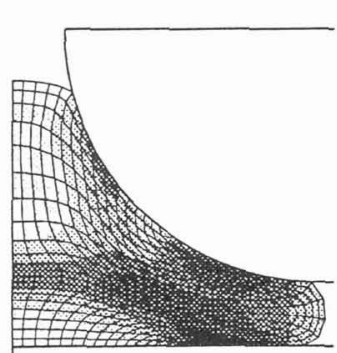

(d)

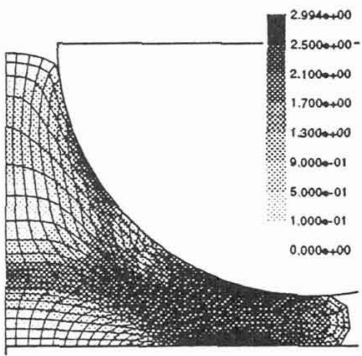

(e)

Fig.7. Axisymmetric hot spike forming. Effective plastic strain distribution at different stages of tool displacement: (a) initial configuration; (b) $\bar{u}=7.6 \mathrm{~mm}$; (c) $\bar{u}=13.0 \mathrm{~mm}$; (d) $\bar{u}=14.4 \mathrm{~mm}$; (e) $\bar{u}=15.0 \mathrm{~mm}$. 


\section{CONCLUDING REMARKS}

A numerical model for bulk metal forming operations which inclucles elastic behaviour is presented. It is numerically unified for both cold (rate-independent) and hot (rate-dependent) temperature regimes and metalurgically sound high temperature interpolation functions are employed. Computational attention has to date been focused on the main mechanical aspects, namely incompressible flow at large elastic-inelastic strains and plastic theories of quasi-static friction. The robust and consistent numerical treatment of the thermo-mechanical theoretical formulations ensures generality and future upgradability of the model. The capabilities of the model to represent physically realistic behaviours are clearly shown in the plane strain benchmark test in which severe localisation occurs, as well as in the three simulations of real industrial processes.

\section{REFERENCES}

[1] ERINGEN A.C., (1975), Continuum Physics, Academic Press, New York

[2] ODEN J.T., (1972), Finite Elements of Nonlinear Continua, McGraw-Hill, New York

[3] KOBAYASHI S., (1984), Thermoviscoplastic analysis of netal forming problems by the finite element method, in Numerical Analysis of Forming Processes, J.F.T. Pittman et.al.(eds.), John Wiley, New York, pp.49-69

[4] ARGYRIS J.H., DOLTSINIS J.St., \& WÜSTENBERG H.W., (1984), Analyses of Thermoplastic Forming natural approach, in Numerical Analysis of Forming Processes, J.F.T. Pitman et.al.(eds.), John Wiley, New York, pp.89-115

[5] RODI $\tilde{C}$ T., (1989), Numerical Analysis of Thermomechanical Processes During Deformations of Metals at High Temperatures, Ph.D. Thesis at University College of Swansea, Wales, U.K.

[6] ARGYRIS J.H. \& DOLTSINIS J.St., (1981), On the natural formulation and analysis of large deformation coupled thermomechanical problems, Comp. Meth. Appl. Mech. Eng., Vol.25, pp.195-253

[7] FJÆR H.G. \& MO A., (1990), ALSPEN-A mathematical model for thermal stresses in direct chill casting of aluminium billets, Metallurgical Transactions $B, \mathrm{Vol} .21 \mathrm{~B}, \mathrm{pp} .1049-1060$

[8] NEDREBERG M.L., (1990), Ph.D. Thesis at University of Oslo, Norway

[9] KIM S.J. \& ODEN J.T., (1984), Generalized Flow Potentials in Finite Elastoplasticity, Int. J. Eng. Sci., Vol.22, pp. 1235-1257

[10] KIM S.J. \& ODEN J.T., (1985), Generalized Flow Potentials in Finite Elastoplasticity, Part II-Examples, Int. J. Eng. Sci., Vol.23, pp.515-530

[11] PERIĆ Dj., OWEN D.R.J. \& HONNOR M., (1992), A model for finite strain elasto-plasticity based on logarithmic strains: Computational issues, Comp. Meth. Appl. Mech. Eng., Vol.94, pp.35-61

[12] PERIĆ Dj. \& OWEN D.R.J., (1991), A model for large deformations of elasto-viscoplastic solids at finite strains: Computational issues, Proceedings of IUTAM Symposium on Finite Inelastic Deformations, Hannover

[13] PERIC Dj., (1992), On consistent stress rates in solid mechanics: Computational implications, Int. J. Num. Meth. Eng., Vol.33, pp.799-817

[14] ODEN J.T. \& MARTINS J.A.C., (1985), Models and Computational Methods for Dynamic Friction Phenomena, Comp. Meth. Appl. Mech. Eng., Vol.52, pp.527-634

[15] AVITZUR B. \& NAKAMURA Y., (1986), Analytical Determination of Friction Resistance as a Function of Normal Load and Geometry of Surface Irregularities, Wear, Vol.107, pp.367-383

[16] FREDERIKSSON B., (1976), Finite Element Solution of Surface Non-linearities in Structural Mechanics with Special Emphasis to Contact and Fracture Mechanics Problems, Comp. \& Struct., Vol.6, pp.281-290

[17] MICHALOWSKI R. \& MROZ Z., (1978), Associated and Non-associated Sliding Rules in Contact Friction Problems, Arch. Mech., Vol.30, pp.259-276

[18] CURNIER A., (1984), A Theory of Friction, Int. J. Solids \& Struct., Vol.20, pp.637-647

[19] SIMO J.C \& ARMERO F., (1992), Geometrically non-linear enhanced strain mixed method and the method of compatible modes, Int. J. Num. Meth. Eng., Vol.33, pp.1413-1449

[20] WRIGGERS P. \& SIMO J.C, (1985), A note on tangent stiffness for fully nonlinear contact problems, Comm. Appl. Num. Meth., Vol.1, pp.199-203

[21] PARISH H., (1989), A consistent tangent stiffness matrix for three-dimensional non-linear contact analysis, Int. J. Num. Meth. Eng., Vol.28, pp.1803-1812

[22] WRIGGERS P., VU VAN T. \& STEIN E., (1989), Finite element formulation of large deformation impactcontact problems with friction, Comp. \& Struct., Vol.37, pp.319-331

[23] HINTON E. \& OWEN D.R.J, (1979), An introduction to finite element computations, Pineridge Press, Swansea

[24] AL-SALEHI F.A.R., FIRBANK T.C. \& LANCASTER P.R., (1973), An experimental determination of the roll pressure distributions in cold rolling, Int. J. Mech. Sci., Vol.15, pp.693-710

[25] LI G.J. \& KOBAYASHI S., (1982), Rigid-plastic finite-element analysis of plane strain rolling, J. Eng. Ind., Vol.104, pp.55-64 